\title{
Phantom based evaluation of CT to CBCT image registration for proton therapy dose recalculation
}

\author{
Guillaume Landry ${ }^{1,2}$, George Dedes ${ }^{1}$, Christoph Zöllner ${ }^{1}$, \\ Josefine Handrack ${ }^{1}$, Guillaume Janssens ${ }^{3}$, Jonathan Orban de \\ Xivry $^{3}$, Michael Reiner ${ }^{2}$, Chiara Paganelli ${ }^{4}$, Marco Riboldi ${ }^{4}$, \\ Florian Kamp ${ }^{5,6}$, Matthias Söhn ${ }^{2}$, Jan J Wilkens ${ }^{5,6}$, \\ Guido Baroni ${ }^{4}$, Claus Belka ${ }^{2}$ and Katia Parodi ${ }^{1}$ \\ 1 Department of Physics, Ludwig-Maximilians-University, Munich, Germany \\ 2 Department of Radiation Oncology, Ludwig-Maximilians-University, Munich, Germany \\ 3 ICTEAM, Université Catholique de Louvain, Louvain-La-Neuve, Belgium \\ 4 Dipartimento di Elettronica Informazione e Bioingegneria, Politecnico di Milano, Milan, Italy \\ 5 Department of Radiation Oncology, Technische Universität München, Klinikum rechts der Isar, \\ Munich, Germany \\ ${ }^{6}$ Physik-Department, Technische Universität München, Garching, Germany
}

\begin{abstract}
The ability to perform dose recalculation on the anatomy of the day is important in the context of adaptive proton therapy. The objective of this study was to investigate the use of deformable image registration (DIR) and cone beam CT (CBCT) imaging to generate the daily stopping power distribution of the patient. We investigated the deformation of the planning CT scan (pCT) onto daily CBCT images to generate a virtual CT (vCT) using a deformable phantom designed for the head and neck $(\mathrm{H} \& \mathrm{~N})$ region.

The phantom was imaged at a planning CT scanner in planning configuration, yielding a pCT and in deformed, treatment day configuration, yielding a reference CT (refCT). The treatment day configuration was additionally scanned at a CBCT scanner. A Morphons DIR algorithm was used to generate a vCT. The accuracy of the vCT was evaluated by comparison to the refCT in terms of corresponding features as identified by an adaptive scale invariant feature transform (aSIFT) algorithm. Additionally, the vCT CT numbers were compared to those of the refCT using both profiles and regions of interest and the volumes and overlap (DICE coefficients) of various phantom structures
\end{abstract}

This is the Accepted Manuscript version of an article accepted for publication in Physics in Medicine \& Biology, 2015, 60, 595-613. IOP Publishing Ltd is not responsible for any errors or omissions in this version of the manuscript or any version derived from it. The Version of Record is available online at: http:// dx.doi.org/10.1088\%2F0031-9155\%2F60\%2F2\%2F595 
were compared. The water equivalent thickness (WET) of the vCT, refCT and pCT were also compared to evaluate proton range differences. Proton dose distributions from the same initial fluence were calculated on the refCT, vCT and $\mathrm{pCT}$ and compared in terms of proton range. The method was tested on a clinical dataset using a replanning CT scan acquired close in time to a CBCT scan as reference using the WET evaluation.

Results from the aSIFT investigation suggest a deformation accuracy of $2-3 \mathrm{~mm}$. The use of the Morphon algorithm did not distort CT number intensity in uniform regions and WET differences between $\mathrm{VCT}$ and refCT were of the order of $2 \%$ of the proton range. This result was confirmed by proton dose calculations. The patient results were consistent with phantom observations. In conclusion, our phantom study suggests the vCT approach is adequate for proton dose recalculation on the basis of CBCT imaging.

Keywords: CBCT, deformable image registration, proton therapy, adaptive radiotherapy, virtual $\mathrm{CT}$

\section{Introduction}

The treatment of head and neck $(\mathrm{H} \& \mathrm{~N})$ cancer has been shown to benefit from increased dose conformity and healthy tissue sparing from intensity modulated photon therapy (IMRT) in terms of reduction of radiation therapy toxicity (Kam et al 2007). This suggests that proton therapy, with its superior dose conformity, may be of benefit to patients undergoing radiation therapy for $\mathrm{H} \& \mathrm{~N}$ lesions. This is supported by intensity modulated proton therapy (IMPT) planning studies reporting improved sparing of organs at risk compared to IMRT (Simone et al 2011, van de Water et al 2011).

However, the increased conformity of IMPT may come at the cost of higher sensitivity to treatment uncertainties, as accurately positioning the Bragg peak is dependent on the accu-racy of the computed tomography (CT) derived 3D stopping power map of the patient. The significant volumetric changes observed during fractionated radiation therapy of $\mathrm{H}$ \& N can-cer (Barker et al 2004) alongside positioning uncertainties have led investigators to recom-mend repeated CT scanning for dose recalculation during IMRT (Wang et al 2010) and more recently for IMPT (Kraan et al 2013). This entails a heavier workload at the CT scanner, additional appointments for the patient and may not capture patient positioning differences between the CT and treatment couches. The use of a CTon-rail, investigated in the context of passively scattered proton therapy of the prostate (Trofimov et al 2011), would offer the optimal data for dose recalculation, although such installations are not common and increase the complexity of the delivery procedure.

The use of cone beam CT (CBCT) imaging at the treatment couch, which is considered for proton therapy setup corrections (Park et al 2009) and currently being introduced by vendors in their products, provides a simpler image acquisition alternative. However CBCT images are of lower quality than those from CT (Stock et al 2009); one major issue is that the relation between CT and CBCT image intensities varies across the field of view (FOV). For example CBCT soft tissue intensities in the upper thorax area are lower than in the neck area. While for photon therapy it may be possible to perform CT number to electron density calibration and achieve acceptable dose calculation accuracy for uncorrected CBCT images (Yang et al 
2007), this has not been demonstrated for protons where CBCT intensity correction to match CT image intensities is most likely required. Preliminary studies have investigated CT num-ber correction for CBCT images (Bentefour et al 2010). It may be possible to obtain accurate stopping power estimates by performing deformable image registration (DIR) between the planning CT and CBCT. This has been explored by several groups in the context of adaptive photon therapy for photon dose recalculation and automatic contour propagation (Zhang et al 2007, Wu et al 2009, Peroni et al 2012, Zhen et al 2012, van Kranen et al 2013, Veiga et al 2014). To the best of our knowledge, CT to CBCT DIR has not been thoroughly evaluated in the context of $\mathrm{H} \& \mathrm{~N}$ proton therapy.

The objective of this work was to perform a phantom study to evaluate CT to CBCT DIR to yield accurate stopping power maps corresponding to the anatomy at treatment time. The use of DIR in this context may be used for two purposes. The first and more straightforward approach consists of providing a tool for clinical decision making by evaluation of the dose distribution of the day. The second approach, which is more complex and demanding of the DIR, consists of accumulating the dose of the day onto the planning CT anatomy. This work targets the first approach, as the evaluation of dose accumulation based on the vector fields obtained from DIR was deemed outside the scope of this study. We investigated DIR in terms of geometrical and CT number accuracy as well as dosimetric accuracy using a deforma-ble phantom designed specifically for $\mathrm{H} \&$ $\mathrm{N}$ anatomical changes. This phantom study has the advantage of providing ground truth against which to compare deformation results. We focused specifically on anatomical changes and for this reason, in this study, translational positioning errors were minimized by rigid registration. In addition to the thorough phantom study, we also present a first application of our method to a real $\mathrm{H} \& \mathrm{~N}$ clinical case exhibiting anatomical changes.

\section{Materials and methods}

\subsection{Phantom}

A phantom specific to $\mathrm{H} \& \mathrm{~N}$ deformations was built for this study. The phantom was designed to simulate the main deformations observed in $\mathrm{H} \& \mathrm{~N}$ patients: weight loss, neck tilt in the anterior-posterior direction and variation of airway diameter due either to tumor volume changes or differences in the patient's position between planning CT and CBCT. The phan-tom, mainly consisting of PMMA, is presented in figure 1 and its components are described in table 1. The two non-PMMA materials, muscle and vertebral column were from CIRS (CIRS Inc, Norfolk, VA). We observed that in patient CT scans, the vertebral column had a CT num-ber distribution centered at $650 \mathrm{HU}$ and the vertebral column material was chosen to approxi-mate this. The phantom was scanned at a planning CT (Toshiba Aquilion 16 LB, Toshiba Medical Systems, the Netherlands) using the clinical protocol for $\mathrm{H} \& \mathrm{~N}$ patients in the plan-ning configuration (no neck tilt, $14.4 \mathrm{~cm}$ diameter, airway diameter of $3 \mathrm{~cm}$, figure $1(a))$ and in treatment day configuration $(2.5$ degree neck tilt, $13 \mathrm{~cm}$ diameter and airway diameter of $2.4 \mathrm{~cm}$, figure $1(b)$ ). Images were reconstructed with $3 \mathrm{~mm}$ slice thickness and $1.074 \mathrm{~mm}$ voxel size in the axial plane. While registration results could potentially be improved by reconstruct-ing images with thinner slices ( 1 or $2 \mathrm{~mm}$ ), we used $3 \mathrm{~mm}$ to be consistent with patient data on which the method is to be applied.

The phantom was subsequently scanned using the on-board CBCT imager of an Elekta Synergy Linac equipped with XVI R4.5 (Elekta, Sweden) in treatment day configuration and reconstructed with $1 \mathrm{~mm}$ isotropic voxel size. We made use of the cinical protocol for $\mathrm{H} \& \mathrm{~N}$ 

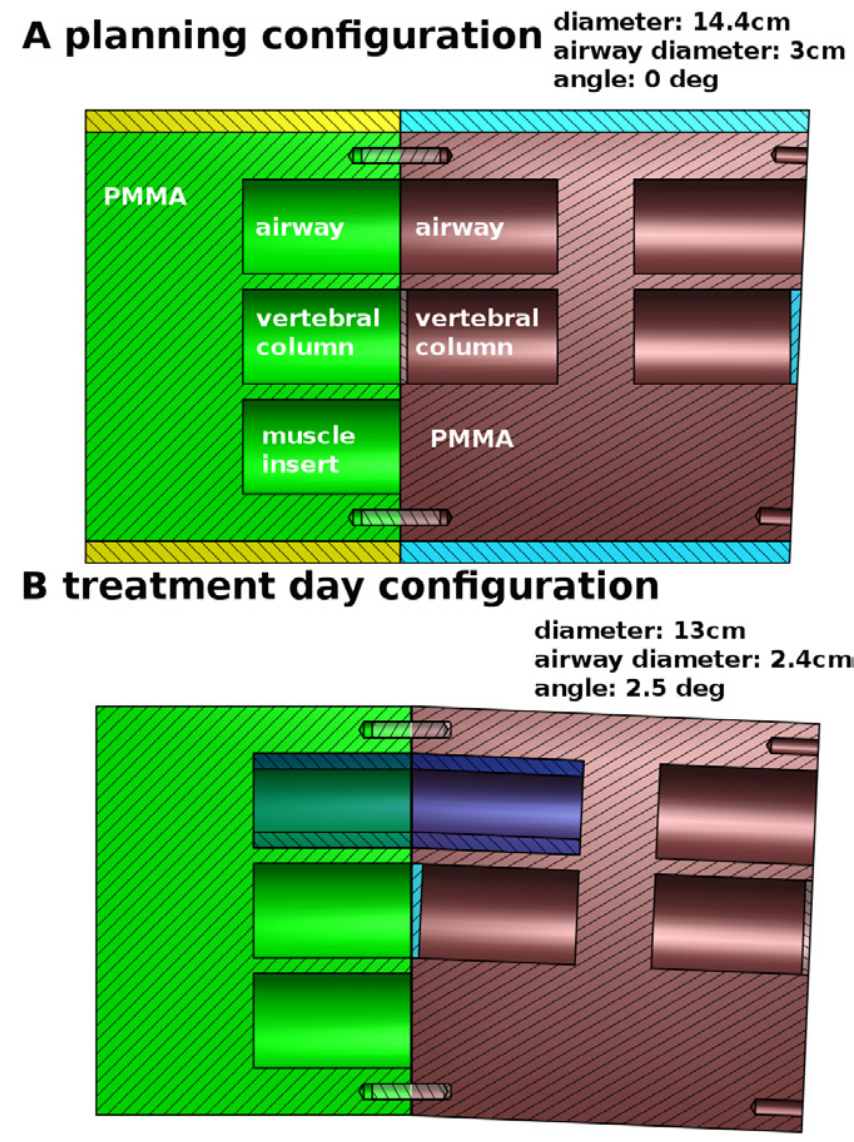

Figure 1. (a) Planning configuration: PMMA deformable phantom modeling $\mathrm{H} \&$ $\mathrm{N}$ anatomical changes. All cavities are $3 \mathrm{~cm}$ in diameter and accommodate $5 \mathrm{~cm}$ long inserts. The phantom diameter as shown is $14.4 \mathrm{~cm}$. The central channels contain vertebral column mimicking inserts, the top channels are kept empty to represent airways and the lower channel contains a muscle mimicking insert for soft tissue contrast. (b) Treatment day configuration: weight loss is modeled by the removal of the yellow and light blue sleeves, reducing the diameter to $13 \mathrm{~cm} .2 .5^{\circ}$ neck tilt is modeled by reversing the red right half of the phantom. Airway diameter variation is modeled by inserting a thin sleeve (dark blue) in both airway channels, reducing the diameter from 3 to $2.4 \mathrm{~cm}$. Two PMMA spacers, one for each angle configuration, are used between the two vertebral column inserts.

patients, using $18.3 \mathrm{mAs}$ at $100 \mathrm{kV}$ with $\mathrm{CTDI}_{\mathrm{vol}}$ of $0.6 \mathrm{mGy}$. No beam shaping filter was used and the S20 collimation was employed.

The treatment day CT scan (refCT) served as ground truth for deformation evaluation. The phantom modification causing the largest volume change from the planning to the treatment day configuration was the removal of the outer ring to reduce the phantom diameter. To assess whether our validation was sensitive to the two other anatomy changes investigated, we included two additional configurations: airway diameter modification only and phantom angle modification only. Table 2 summarizes all the configurations. While tests were performed for each configuration, in this paper we focus on configuration 3 as it represents the combination of all effects. 
Table 1. Characteristics of the phantom components. The muscle and solid bone inserts are from CIRS (CIRS Inc, Norfolk, VA). The CT numbers reported here are estimated from the planning CT scan using a circular region of interest in a single slice.

\begin{tabular}{llr}
\hline component & material & CT number $(\mathrm{HU})$ \\
\hline phantom body & PMMA & $120 \pm 5$ \\
fat ring & PMMA & $120 \pm 5$ \\
muscle insert & Muscle 062A-27 & $53 \pm 5$ \\
vertebral column & Solid bone $\left(400 \mathrm{mg} \mathrm{HA}^{-1}\right)$ & $590 \pm 6$ \\
Airway & air & $-1048 \pm 6$ \\
\hline
\end{tabular}

Table 2. Phantom configurations studied in this study. Configuration 3 is the one presented in figure $1(b)$.

\begin{tabular}{ll}
\hline configuration & anatomical change modelled \\
\hline 1 & airway diameter reduction \\
2 & neck tilt \\
3 & Airway diameter reduction, neck tilt and weight loss \\
\hline
\end{tabular}

\subsection{Registration}

The REGGUI DIR package was employed to perform 3D image registration between plan-ning CT (pCT) and CBCT images (Janssens et al 2011) using an automated workflow. This tool was previously evaluated in the context of dose accumulation based on MV CBCT images (Janssens et al 2009). When performing DIR with REGGUI, images are required to be in the same coordinate system and have the same grid spacing. In this work we opted for the CBCT coordinate system and grid spacing as the CBCT has a higher information density than the $\mathrm{pCT}$. A rigid registration limited to translations was employed to align the pCT to the CBCT based on the sum of the squared intensity differences. Rotations were not permitted to repli-cate the limited degrees of freedom of clinical alignment procedures. As a result of rigid regis-tration in REGGUI, the pCT $(1.074 \times 1.074$ $\times 3 \mathrm{~mm})$ was resampled to the CBCT image grid $(1 \times 1 \times 1 \mathrm{~mm})$ using linear interpolation. DIR of the resampled pCT to the CBCT was subse-quently performed using the REGGUI implementation of the Morphons algorithm (Knutsson and Andersson 2005, Wrangsjo et al 2005) using 8 scales with 10 iterations for the 6 coarsest scales and 2 iterations for the last two higher resolution scales (the highest resolution is the CT resolution). Accumulation of the deformation field at each scale was performed with a Gaussian regularization filter with a standard deviation of 1.5 voxels. The Morphons algorithm is suitable for CT to CBCT DIR as it is based on a local phase metric and is thus insensitive to intensity differences between the $\mathrm{pCT}$ and $\mathrm{CBCT}$. The procedure yielded a deformed $\mathrm{pCT}$ in the coordinate system and grid spacing of the CBCT. We called this image the virtual CT, as in Peroni et al (Peroni et al 2012). As this image is in the CBCT coordinate system, we add the CBCT subscript $\left(\mathrm{vCT}_{\mathrm{CBCT}}\right)$. In general, images in the $\mathrm{CBCT}$ coordinate system and grid spacing have the CBCT subscript in this paper. The corresponding refCT was aligned with the CBCT and resampled to the $\mathrm{CBCT}$ grid spacing as well using a rigid registration, this time allowing for rotations, yielding the $\operatorname{refCT}_{\mathrm{CBCT}}$, for direct evaluation of the $\mathrm{vCT}_{\mathrm{CBCT}}$. Rotations were allowed to achieve an optimal reference image.

The vector field resulting from the Morphons DIR was in the coordinate system and grid spacing of the CBCT. To apply it to the original $\mathrm{pCT}$ we transferred the vector field to the 
pCT coordinate system using the inverse of the rigid translation registration and resampled it to the original pCT grid by linearly interpolating the components of the deformation vectors. Applying this vector field to the original pCT yielded a virtual $\mathrm{CT}$ in the coordinate system and grid spacing of the $\mathrm{pCT}\left(\mathrm{vCT}_{\mathrm{pCT}}\right)$. In general in this paper, images in the pCT coordinate system and grid spacing have the pCT subscript. The $\mathrm{vCT}_{\mathrm{pCT}}$ can be used interchangeably with the $\mathrm{pCT}_{\mathrm{pCT}}$ to evaluate the dosimetric impact of anatomical changes by recalculating the dose distribution of a plan. To evaluate the $\mathrm{vCT}_{\mathrm{pCT}}$ the refCT was also rigidly aligned to it, allowing rotations, yielding a refCT $\mathrm{TCT}_{\mathrm{pC}}$.

\subsection{Scale invariant feature transform evaluation}

The scale invariant feature transform (SIFT) (Lowe 1999, Lowe 2004, Cheung and Hamarneh 2009) was employed to evaluate DIR accuracy. The SIFT method is capable of automati-cally extracting corresponding features between pairs of images. The methods described in Paganelli et al (Paganelli et al 2013a) and implemented in plastimatch (Shackleford et al 2010) were employed to compare CT and CBCT images. However, the high homogeneity of the phantom materials led to a very low number of identified features using the conventional SIFT algorithm.

For this reason, we employed a variant of the SIFT method making use of adaptive con-trast, which allows for a more efficient feature identification at the cost of a larger number of outliers and was shown to report similar feature distances as the original method (Paganelli et al 2013b). This method, aSIFT, was employed in this work, as it proved better suited for phantom evaluation due to the higher CT number uniformity of plastic materials.

We defined outliers in a similar manner as in Paganelli et al (Paganelli et al 2013b). Feature matches with distances outside a range defined by the 25 th and 75 th percentiles [above 75th +0.5 (75th-25th) and below 25th-0.5 (75th-25th)] were considered outliers. It was observed that the algorithm identified matching features along the phantom or inserts surfaces for which large distances in the direction of the phantom's axis of radial symmetry were present. For this reason, we implemented a $Z$ threshold $\mathrm{T}_{Z}$ of $6 \mathrm{~mm}$ to further remove outliers. $T_{Z}$ was chosen to be larger than any deformation expected from the phantom in the $\mathrm{Z}$ direction and is also twice the rigid registration error of $\sim 3 \mathrm{~mm}$ (or the planning CT slice thickness).

\subsection{Deformation accuracy evaluation}

The registration accuracy of the phantom study was evaluated by inter-comparing the $\mathrm{pCT}_{\text {СвСт }}, \mathrm{vCT}_{\mathrm{CBCT}}$, refCT $\mathrm{CBCT}_{\text {and }} \mathrm{CBCT}_{\mathrm{CBCT}}$ using the aSIFT algorithm for a total of 6 comparisons. Furthermore, each insert (or airway) of the phantom was segmented using a region-growing algorithm applied to the $\mathrm{pCT}_{\mathrm{CBCT}}, \mathrm{vCT}_{\mathrm{CBCT}}$ and $\operatorname{refCT}_{\mathrm{CBCT}}$. Contour volumes and DICE coef-ficients between corresponding contours were estimated. To evaluate the accuracy of proton range estimation, the water equivalent thickness (WET) was calculated for projections at $0^{\circ}, 90^{\circ}$ and $180^{\circ}$ starting from the phantom's surface down to a normal plane coinciding with the central axis of the phantom and to a second plane beyond the distal end of the phantom. To avoid confounding effects with the $180^{\circ}$ direction, the table CT numbers were cropped to -1024 . The $2 \mathrm{D}$ WET distributions from the $\mathrm{pCT}_{\mathrm{CBCT}}$ and

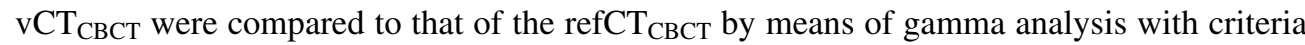
$2 \mathrm{~mm} / 2 \mathrm{~mm}$ WET. We employed a signed-gamma implementation (Persoon et al 2011). The tests mentioned above were per-formed for configurations 1,2 and 3. 


\subsection{Dosimetric evaluation}

The abovementioned tests were performed in the coordinate system and grid spacing of the CBCT. To test the final $\mathrm{vCT}_{\mathrm{pCT}}$ in the coordinate system and grid spacing of the $\mathrm{pCT}$, proton dose calculations were employed. We optimized proton dose distributions using single IMPT beams at $0^{\circ}, 90^{\circ}$ and $180^{\circ}$ delivering a uniform $2 \mathrm{~Gy}$ dose to the vertebral column inserts using the refCT $\mathrm{pCT}_{\mathrm{pC}}$ (rigidly aligned to the $\mathrm{pCT}$ ). A comparison based on the refCT $\mathrm{pCT}_{\mathrm{pC}}$ was chosen as it represents the anatomy of the day which we aim at estimating using the $\mathrm{vCT}_{\mathrm{pCT}}$. To avoid confounding effects with the $180^{\circ}$ beam the table CT numbers were cropped to -1024 . The dose distributions were optimized using an extension of the CERR (Deasy et al 2003) software package by Schell and Wilkens (Schell and Wilkens 2010). The resulting proton fluences were re-projected on the $\mathrm{pCT}_{\mathrm{pCT}}$ and $\mathrm{vCT}_{\mathrm{pCT}}$ using a Geant4 (Agostinelli et al 2003) Monte Carlo (MC) dose calculation engine. The dose volume histograms (DVH) from dose distribu-tions obtained using the $\operatorname{refCT}_{\mathrm{pCT}}, \mathrm{vCT}_{\mathrm{pCT}}$ and $\mathrm{pCT}_{\mathrm{pCT}}$ were compared in terms of DVH sta-tistics, $D_{90}$, as well as the proton range as defined as the distance between the phantom surface and the distal $80 \%$ isodose. 2D proton range maps in beam-eye-view (BEV) from the $\mathrm{pCT}_{\mathrm{pCT}}$, $\mathrm{refCT}_{\mathrm{pCT}}$ and $\mathrm{vCT}_{\mathrm{pCT}}$ were compared using gamma evaluation with criteria of $2 \mathrm{~mm} / 2 \mathrm{~mm}$ Range. The dosimetric evaluation was only performed on configuration 3 .

\subsection{Patient data}

To verify that the DIR workflow developed for the phantom is valid for clinical applications, the imaging data of a $\mathrm{H} \& \mathrm{~N}$ cancer patient undergoing photon IMRT was used in this work. The dataset contained a pCT, a CBCT scan acquired 50 days after the pCT and a replan-ning CT (rpCT) acquired a day after the CBCT scan. The same procedure that was used for the phantom was employed here to generate a patient $\mathrm{vCT}_{\mathrm{CBCT}}$ as well as a rigidly regis-tered $\mathrm{rpCT}_{\mathrm{CBC}}$. The already described WET evaluation was applied to the patient data using the $0^{\circ}$ direction and integrating to the patient midplane. Comparison of the $\mathrm{pCT}_{\mathrm{CBCT}}$ versus $\mathrm{rpCT}_{\mathrm{CBCT}}$ and $\mathrm{vCT}_{\mathrm{CBCT}}$ versus $\mathrm{rpCT}_{\mathrm{CBCT}}$ was performed as described for the phantom. We made use of the gamma evaluation as the patient is heterogeneous in the superior inferior direction, as opposed to the phantom. Slight errors in the rigid registration of the $\mathrm{pCT} / \mathrm{rpCT}$ to the $\mathrm{CBCT}$ can thus have a large impact on the WET difference distributions. For this reason we relaxed the distance to agreement criterion to $3 \mathrm{~mm}$, which is the pCT slice thickness, and kept the WET to agreement at $2 \mathrm{~mm}$.

\section{Results}

Unless stated otherwise the results presented here are for configuration 3.

\subsection{Registration}

Figure 2 presents the $\mathrm{pCT}_{\mathrm{CBCT}}$ in planning configuration, the refCT $\mathrm{CBCT}_{\mathrm{C}}$ and $\mathrm{CBCT}_{\mathrm{CBCT}}$ in treatment day configuration as well as the $\mathrm{vCT}_{\mathrm{CBCT}}$ resulting from DIR of the $\mathrm{pCT}$ to the $\mathrm{CBCT}$. Figure 2 shows the difference in phantom diameter, angle and airway diameter between the treatment day configuration and the planning configuration. We observe that the $\mathrm{vCT}_{\mathrm{CBCT}}$ diameter has been reduced; however the air gap between the phantom body and fat ring caused by an imperfect fit has not been eliminated by the DIR. The reverse situation was observed in the airway where the refCT $\mathrm{CBCT}_{\mathrm{C}}$ exhibits an air gap due to the imperfect fit of the airway 


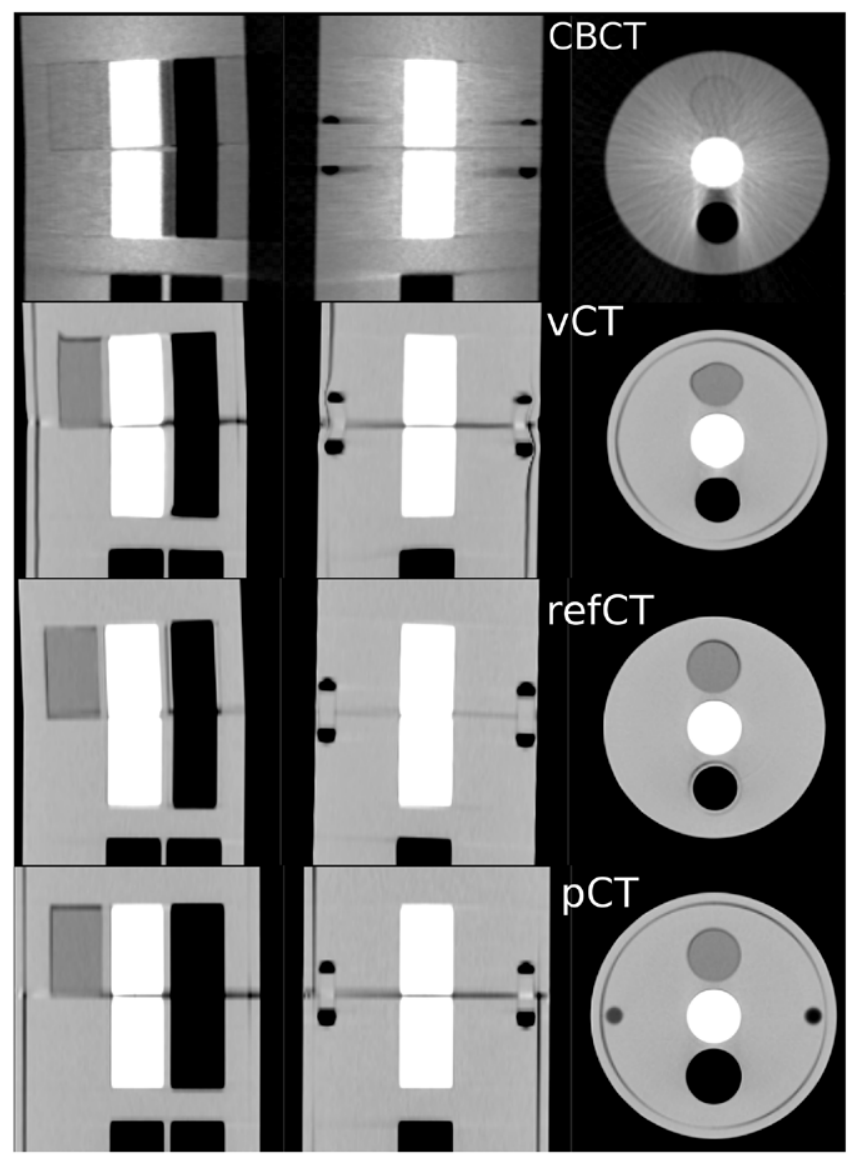

Figure 2. $\mathrm{CT}$ scans of the phantom in treatment day configuration $\left(\mathrm{CBCT}_{\mathrm{CBCT}}\right.$, refCT $\mathrm{CBCT}_{\mathrm{C}}$ ) and in planning configuration $\left(\mathrm{pCT}_{\mathrm{CBCT}}\right)$. The $\mathrm{vCT}_{\mathrm{CBCT}}$ is the result of DIR of the pCT to the CBCT.

sleeve which is not visible in the $\mathrm{vCT}_{\mathrm{CBCT}}$. The agreement between the $\mathrm{vCT}_{\mathrm{CBCT}}$ and the ref$\mathrm{CT}_{\mathrm{CBCT}}$ as well as the difference between the $\mathrm{pCT}_{\mathrm{CBCT}}$ and refCT $\mathrm{CBCT}_{\mathrm{CBC}}$ are better visualized in the checkerboard representation of figure 3 . There is good agreement between $\mathrm{vCT}_{\mathrm{CBCT}}$ and refCT $\mathrm{CBCT}_{\mathrm{C}}$ for the phantom outer contour, airways and vertebral column. However the muscle insert in the $\mathrm{vCT}_{\mathrm{CBCT}}$ has been distorted, most likely due to the low $\mathrm{CT}$ number contrast between this insert and PMMA. It is questionable whether this situation would arise in a patient geometry given the higher number of gradients found in those images. The distortion of the muscle insert raises questions as to the use of the deformation fields to accumulate dose in a reference time point such as the pCT. However an investigation of dose accumulation was beyond the scope of this paper. We observed a misalignment in the $Z$ direction resulting from the rigid deformation of the $\mathrm{pCT}$ to the $\mathrm{CBCT}$. This misalignment is of the order of the slice thickness and could be due to the different geometries being aligned. While manual correction of this misalignment is straightforward this was not done as we aimed at evaluating the complete automatic workflow. Furthermore, the DIR performed following rigid registration corrects for residual shifts. 


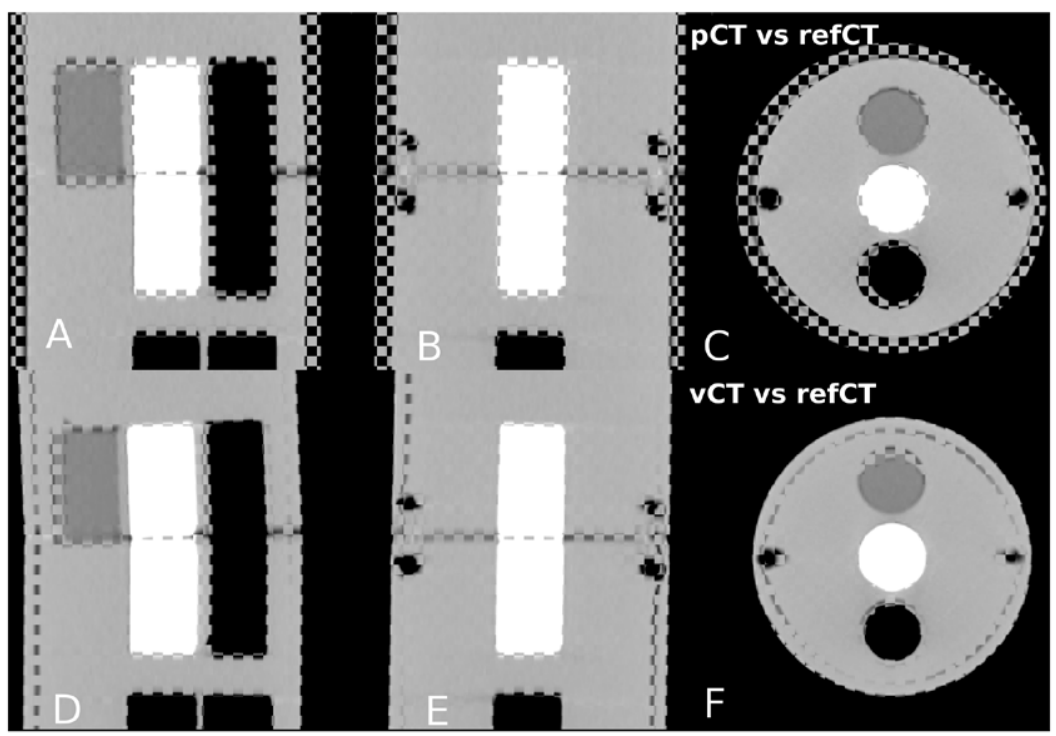

Figure 3. (a)-(c) Checkerboard comparison of the $\mathrm{pCT}_{\mathrm{CBCT}}$ and refCT $\mathrm{CBCT}_{\text {. }}(d)-(f)$ Comparison of the $\mathrm{vCT}_{\mathrm{CBCT}}$ and refC $\mathrm{T}_{\mathrm{CBCT}}$.

\subsection{Deformation accuracy evaluation}

Figure 4 presents the results of the aSIFT evaluation for the 6 possible image intercompari-sons using $T_{\mathrm{z}}=6 \mathrm{~mm}$. We observed that the median distance between corresponding features was lowered when comparing the $\mathrm{vCT}_{\mathrm{CBCT}}$, $\operatorname{refCT}_{\mathrm{CBCT}}$ and $\mathrm{CBCT}_{\mathrm{CBCT}}$ together, indicating that our registration algorithm yielded a $\mathrm{vCT}_{\mathrm{CBCT}}$ which agreed better with the $\mathrm{CBCT}_{\mathrm{CBCT}} /$ refCT $_{\mathrm{CBCT}}$ than the $\mathrm{pCT}_{\mathrm{CBCT}}$. The median distances are of the order of $2-3 \mathrm{~mm}$, which is comparable to the planning CT resolution of $1.074 \mathrm{~mm}$ in the axial plane and $3 \mathrm{~mm}$ in the scan direction. Interpolating the CT to the CBCT coordinate system does not improve its resolution, however the fact that the phantom is homogenous in the $Z$ direction means that for homogeneous parts of the phantom this interpolation should yield similar values as a higher resolution reconstruction. This is however not true at edges, where most features are detected. It is thus likely that our results are limited by a combination of the precision of the aSIFT algorithm and the slice thickness used. The median feature distances for the $\mathrm{vCT}_{\mathrm{CBCT}}$ versus $\operatorname{refCT}_{\mathrm{CBCT}}$ and

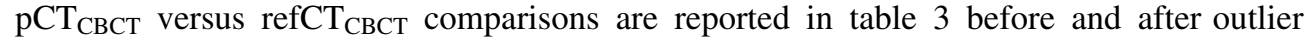
removal. A Wilcoxon rank sum test between the feature distances of the $\mathrm{vCT}_{\mathrm{CBCT}}$ versus refCT $\mathrm{T}_{\mathrm{CBCT}}$ and $\mathrm{pCT}_{\mathrm{CBCT}}$ versus refCT $\mathrm{T}_{\mathrm{CBCT}}$ after outlier removal yielded $\mathrm{p}<0.01$. The aSIFT algorithm yielded matching features between the $\mathrm{vCT}_{\mathrm{CBCT}}$ and refCT $\mathrm{TBCT}_{\mathrm{CBC}} / \mathrm{CBCT}_{\mathrm{CBCT}}$ which exhibited large differences in their $Z$ positions, hence the need for a $T_{\mathrm{Z}}$ rejection. This is illustrated in figure 5 where accepted and rejected features are shown.

Figure 6 presents the results of the aSIFT evaluation between the pCT $_{\text {СBCT }}$ versus ref- $\mathrm{CT}_{\mathrm{CBCT}}$ and $\mathrm{vCT}_{\mathrm{CBCT}}$ versus refCT $_{\mathrm{CBCT}}$ for all configurations. As expected configuration 3 shows the largest difference between $\mathrm{pCT}_{\mathrm{CBCT}}$ and $\mathrm{vCT}_{\mathrm{CBCT}}$, however the $\mathrm{vCT}_{\mathrm{CBCT}}$ shows lower median feature distances than the $\mathrm{pCT}_{\mathrm{CBCT}}$ for configurations 1 and 2 as well.

Figure 7 presents profiles across the phantom comparing the $\mathrm{vCT}_{\mathrm{CBCT}}$, $\operatorname{refCT}_{\mathrm{CBCT}}$ and $\mathrm{pCT}_{\text {СВСт }}$. We observed good geometrical agreement between $\mathrm{vCT}_{\mathrm{CBCT}}$ and refCT $\mathrm{T}_{\mathrm{CBCT}}$ and no noticeable degradation of CT number accuracy was observed in uniform regions. A discrepancy 


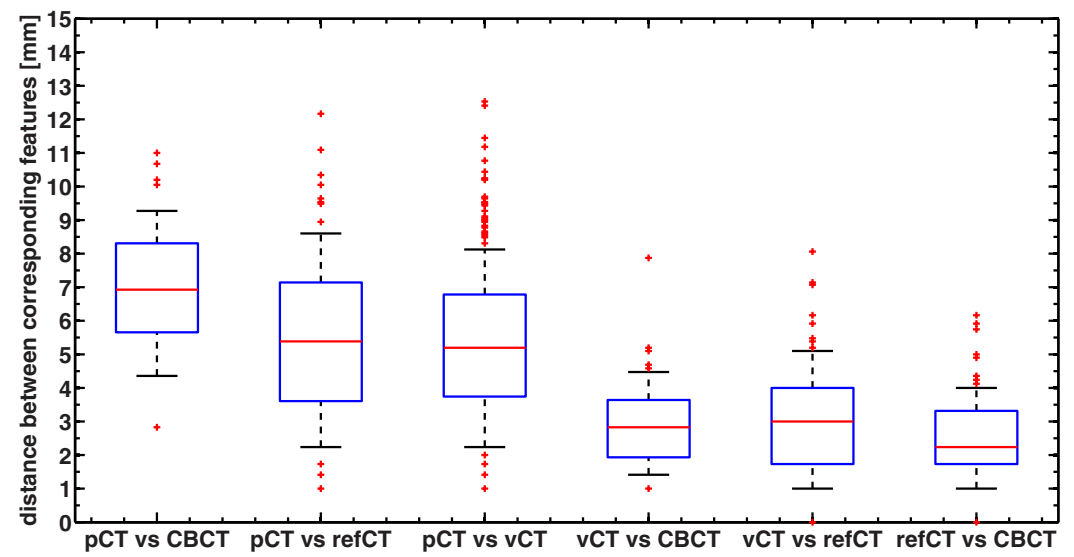

Figure 4. Boxplot of the distance between corresponding features for the 6 comparisons investigated after applying $T_{\mathrm{Z}}=6 \mathrm{~mm}$. The horizontal line represents the median distance; the blue box represents the 25th and 75th percentiles and the whiskers extend to the most extreme data point not considered an outlier (plotted as red crosses). The median calculated here includes outliers, as opposed to table 3 . Images were in the CBCT coordinate system.

Table 3. Median and interquartile range of distances of corresponding features before and after outlier removal for the $\mathrm{pCT}_{\mathrm{CBCT}}$ versus refCT $\mathrm{T}_{\mathrm{CBCT}}$ and $\mathrm{vCT}_{\mathrm{CBCT}}$ versus refCT $\mathrm{T}_{\mathrm{CBCT}}$ comparisons. The number of corresponding features is also reported as well as their anatomical location based on CT number thresholds.

\begin{tabular}{|c|c|c|}
\hline & $\mathrm{pCT}$ versus refCT & vCT versus refCT \\
\hline & \multicolumn{2}{|c|}{$\begin{array}{l}\text { median distance between } \\
\text { corresponding features }(\mathrm{mm})\end{array}$} \\
\hline all features & $21 \pm 35$ & $15 \pm 25$ \\
\hline \multirow[t]{2}{*}{ after outlier removal } & $5.1 \pm 2.9$ & $2.4 \pm 1.9$ \\
\hline & \multicolumn{2}{|c|}{ number of features } \\
\hline all features & 446 & 796 \\
\hline \multirow[t]{2}{*}{ after outlier removal } & 94 & 154 \\
\hline & \multicolumn{2}{|c|}{ number of features by location } \\
\hline CT number $>200$ & 23 & 26 \\
\hline$-200<$ CT number $<200$ & 58 & 77 \\
\hline CT number $<-200$ & 13 & 52 \\
\hline
\end{tabular}

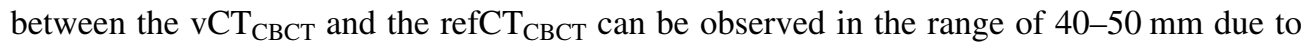
the deformation of the muscle insert in the $\mathrm{vCT}_{\mathrm{CBCT}}$. A second discrepancy is observed between $110-120 \mathrm{~mm}$ due to the imperfect fit of the airway sleeve in the refCT ${ }_{\mathrm{CBCT}}$ which caused a narrow air gap. Table 4 presents the volumes of the automatically segmented inserts and their DICE coefficients between $\mathrm{vCT}_{\mathrm{CBCT}} / \mathrm{refCT}_{\mathrm{CBCT}}$ and $\mathrm{pCT}_{\mathrm{CBCT}} / \mathrm{refCT}_{\mathrm{CBCT}}$. Good agreement was observed between the $\mathrm{vCT}_{\mathrm{CBCT}}$ and $\operatorname{refCT}_{\mathrm{CBCT}}$, with DICE coefficients between 0.83 to 0.99 , an improvement from $\mathrm{pCT}_{\mathrm{CBCT}}$ versus refCT $\mathrm{CBCT}_{\mathrm{T}}$. The worst performance in term of DICE coefficients was the muscle insert which was distorted by the deformation. The DICE 

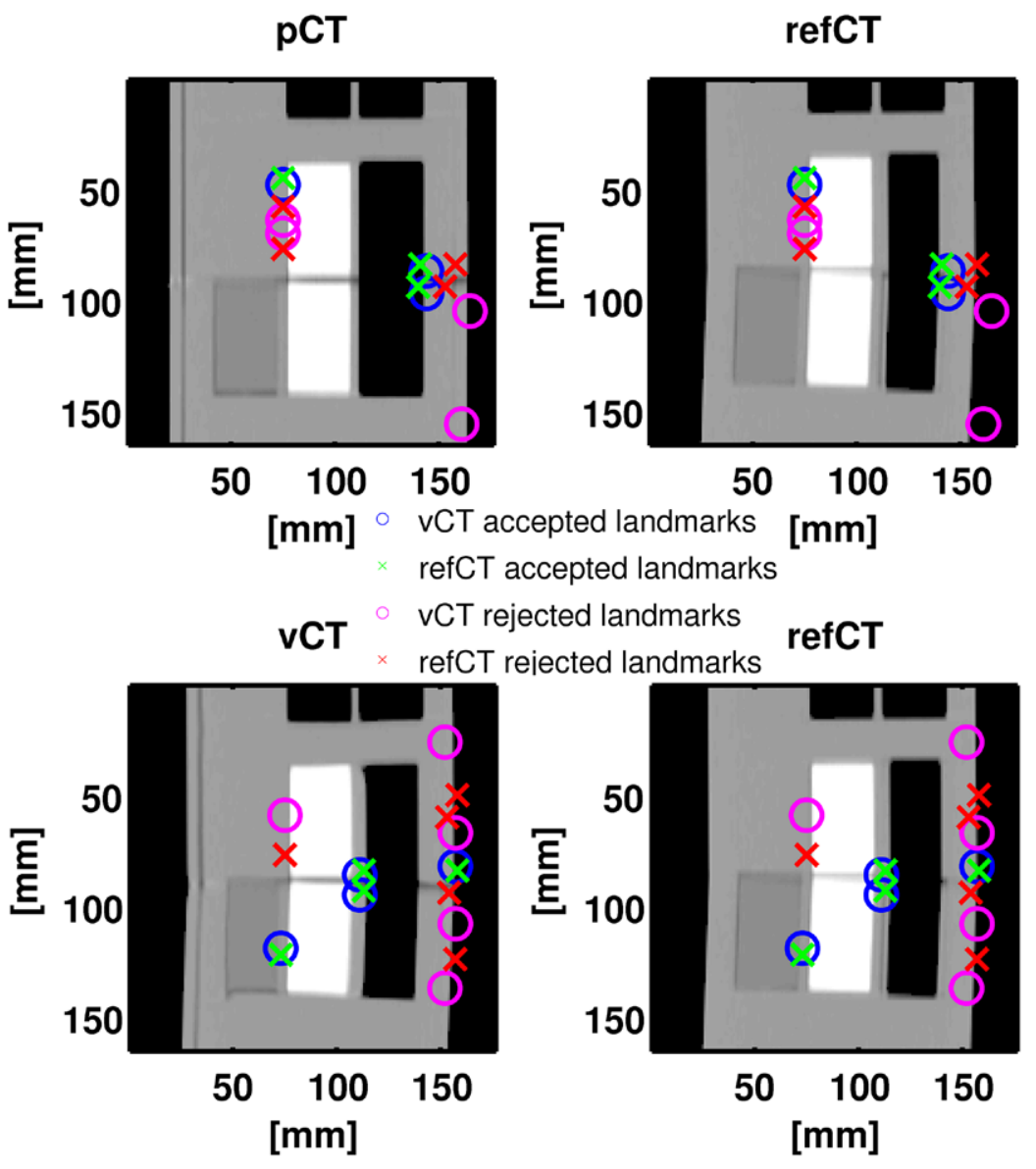

Figure 5. (Top) Corresponding features plotted simultaneously on the $\mathrm{pCT}_{\mathrm{CBCT}}$ and refCT $\mathrm{CBCT}_{\mathrm{CBC}}$ with outliers indicated in red/purple while accepted features are in green/ blue. (Bottom) Corresponding features plotted simultaneously on the $\mathrm{vCT}_{\mathrm{CBCT}}$ and $\operatorname{refCT}_{\mathrm{CBCT}}$.

coefficients for the vertebral column inserts from the $\mathrm{pCT}_{\mathrm{CBCT}}$ versus refC $\mathrm{T}_{\mathrm{CBCT}}$ comparison, which had constant volume, were below 0.9 due to the misalignment from the rigid registration between $\mathrm{pCT}_{\mathrm{CBCT}}$ and refC $\mathrm{T}_{\mathrm{CBCT}}$ which can be observed in figure 3 . However the volumes of the vertebral column inserts were consistent across all scans. Insert volumes were lower than the nominal $35.3 \mathrm{~cm}^{3}$ due to imperfect insert segmentation. The errors on the volumes are consistent with an underestimation of the insert radius by $1 \mathrm{~mm}$ which is of the order of the CBCT voxel size. When considering configurations 1 and 2 similar results were obtained, however in those cases the volume of the muscle insert was preserved in the $\mathrm{vCT}_{\mathrm{CBCT}}$.

Figure 8 presents the WET evaluation at $0^{\circ}, 90^{\circ}$ and $180^{\circ}$ and mid-depth when the $\mathrm{vCT}_{\mathrm{CBCT}}$ and refCT $\mathrm{CBCT}_{\mathrm{C}}$ are compared. The results of the WET evaluation are summarized in table 5. For the $\triangle \mathrm{WET}$ evaluation of table 5 the rows of pixels where the beam was tangential to the phantom surface ( 2 pixel rows on either side of the phantom) were excluded from the analysis as the very low WET values gave relative WET differences larger than $100 \%$. Additionally, pixels with WET differences larger than $40 \mathrm{~mm}$ were considered 


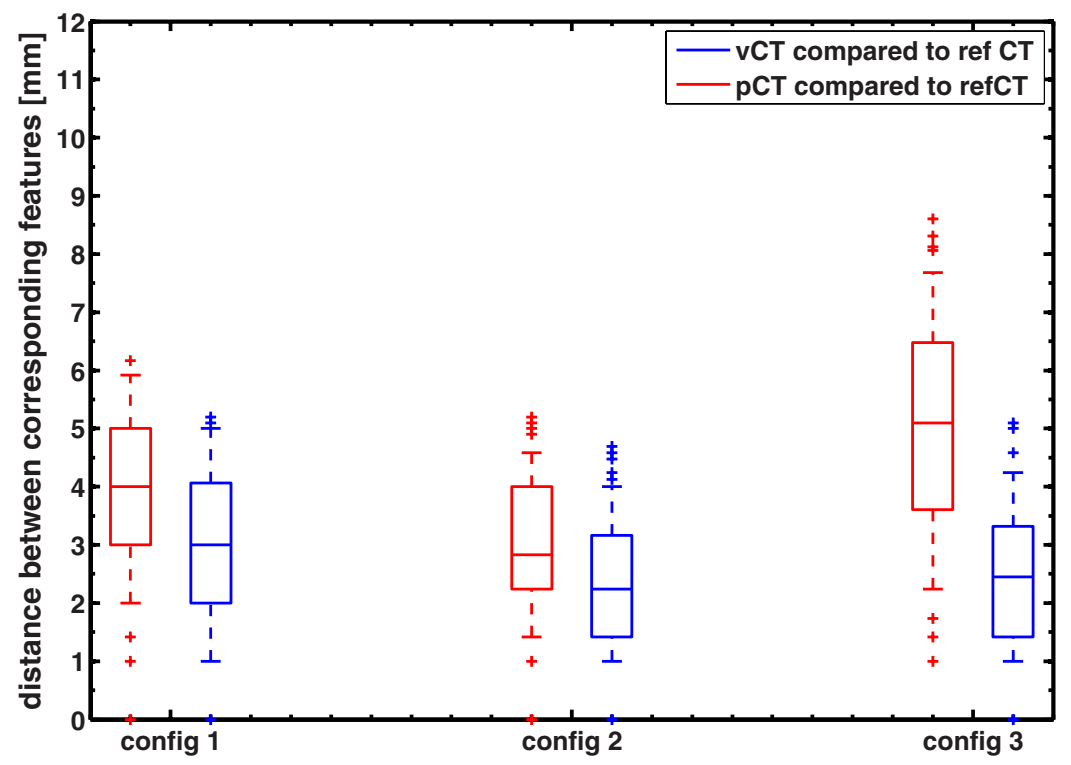

Figure 6. Boxplot of the distance between corresponding features for the 3 configurations. The horizontal line represents the median distance; the blue box represents the 25th and 75th percentiles and the whiskers extend to the most extreme data point not considered an outlier (plotted as red crosses).

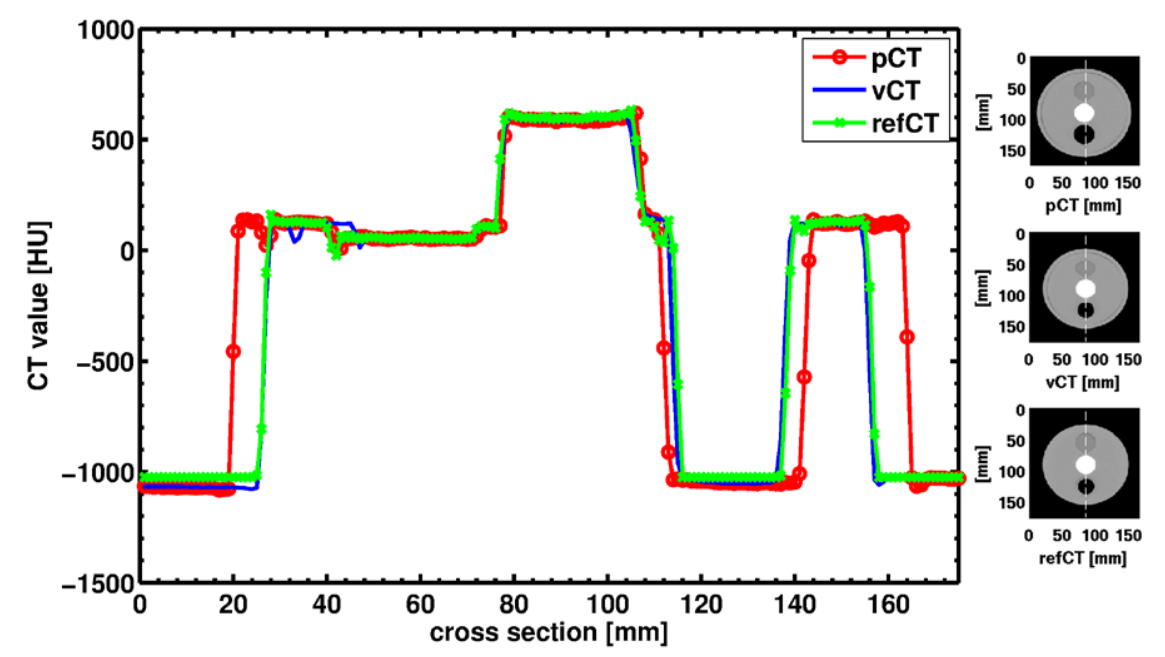

Figure 7. CT number profiles along the white dashed line indicated on the right for the $\mathrm{pCT}_{\mathrm{CBCT}}, \mathrm{vCT}_{\mathrm{CBCT}}$ and refCT $\mathrm{CBCT}$.

outliers. For the gamma evaluation all pixels were considered. We observed that few pixels pass the gamma evaluation and that the average WET difference was large when comparing the $\mathrm{pCT}_{\mathrm{CBCT}}$ and the refCT $\mathrm{CBCT}_{\mathrm{CB}}$, as can be expected from the removal of the outer ring, which is $7 \mathrm{~mm}$ thick. Comparing the $\mathrm{vCT}_{\mathrm{CBCT}}$ and refCT $\mathrm{CBCT}_{\mathrm{C}}$ yielded a high level of 
Table 4. Volumes of phantom components estimated from the $\mathrm{pCT}_{\mathrm{CBCT}}$, $\mathrm{vCT}_{\mathrm{CBCT}}$ and $\operatorname{refCT}_{\mathrm{CBCT}}$ as well as the DICE coefficient comparing the components from the $\mathrm{pCT}_{\mathrm{CBCT}}$ versus refCT $\mathrm{CBCT}_{\mathrm{CBC}}$ and $\mathrm{vCT}_{\mathrm{CBCT}}$ versus refCT $\mathrm{CBCT}_{\text {. }}$

\begin{tabular}{lllllll}
\hline & \multicolumn{3}{c}{ volume $\left(\mathrm{cm}^{3}\right)$} & & \multicolumn{2}{c}{ DICE coefficient } \\
\cline { 2 - 3 } Component & pCT & vCT & refCT & & $\begin{array}{l}\text { pCT versus } \\
\text { refCT }\end{array}$ & $\begin{array}{l}\text { vCT versus } \\
\text { refCT }\end{array}$ \\
\hline vertebral column 1 & 30.6 & 30.6 & 31.6 & & 0.83 & 0.95 \\
vertebral column 2 & 30.4 & 30.8 & 31.0 & & 0.87 & 0.95 \\
airway & 73.2 & 47.0 & 46.2 & & 0.75 & 0.94 \\
muscle & 30.4 & 25.4 & 32.0 & & 0.89 & 0.83 \\
body & 2755 & 2230 & 2240 & & 0.89 & 0.99 \\
\hline
\end{tabular}
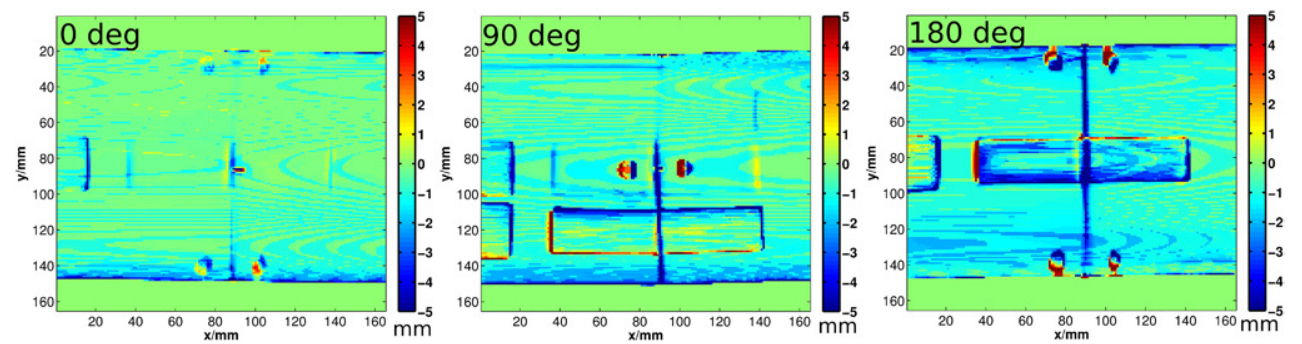

Figure 8. WET difference maps in mm between the $\mathrm{vCT}_{\mathrm{CBCT}}$ and refCT $\mathrm{CBCT}_{\mathrm{CBC}}\left(\mathrm{WET}_{\mathrm{vCT}}-\right.$ $\left.\mathrm{WET}_{\text {refCT }}\right)$ at $0^{\circ}, 90^{\circ}$ and $180^{\circ}$ and mid phantom depth.

passing pixels $(>98 \%$ ) and mean WET differences of the order of $-1 \mathrm{~mm}$ at the center of the phantom and $-2 \mathrm{~mm}$ when traversing the whole phantom. These differences correspond to about -2 to $-2.5 \%$ of the WET. Most of the WET differences were observed at the border of the phantom as seen in figure 8 . This is caused by an imperfect correction of the phantom diameter following the removal of the outer ring. Such an effect is visible in in the vCT ver-sus refCT comparison of figure 3(f) at the right and bottom edges of the phantom. The slight mismatch is sufficient to cause large WET differences at edges; however these WET errors would not appear in dose distributions for beams covering the central part of the phantom. In that case the error would be limited to the mismatch of phantom diameter, which is of the order of $1 \mathrm{~mm}$.

We observed that the WET evaluation was not particularly sensitive to the smaller changes of configurations 1 and 2 as opposed to configuration 3 . The $\mathrm{vCT}_{\mathrm{CBCT}}$ results were consistent across configurations.

\subsection{Dosimetric evaluation}

We observed that the distal fall off of the dose distributions agreed well between $\mathrm{vCT}_{\mathrm{pCT}}$ and refCT $\mathrm{pCT}_{\mathrm{pC}}$. As expected from the anatomical changes, this was not the case for the $\mathrm{pCT}_{\mathrm{pCT}}$ where an under-dosage of the target was observed.

Figure 9 presents the range difference maps corresponding to the $0^{\circ}, 90^{\circ}$ and $180^{\circ}$ beams We observe that range differences are reduced when using the $\mathrm{vCT}_{\mathrm{pCT}}$ instead of the $\mathrm{pCT}_{\mathrm{pCT}}$. Since range is calculated from the phantom surface, the $7 \mathrm{~mm}$ difference caused by the weight 
Table 5. WET comparison between $\mathrm{pCT}_{\mathrm{CBCT}} / \mathrm{refCT}_{\mathrm{CBCT}}$ and $\mathrm{vCT}_{\mathrm{CBCT}} /$ refCT $\mathrm{CBCT}_{\mathrm{C}}$ at $0^{\circ}, 90^{\circ}$ and $180^{\circ}$ at two phantom depths. Mean WET difference $\left(\mathrm{WET}_{\mathrm{vCT}}\right.$ or $\left.\mathrm{pCT}-\mathrm{WET}_{\text {refCT }}\right)$ as well as standard deviation and \% pixels passing a $2 \mathrm{~mm} / 2 \mathrm{~mm}$ WET gamma evaluation are presented.

\begin{tabular}{|c|c|c|c|c|}
\hline angle (degree) & phantom depth & $\begin{array}{l}\% \text { passing } \\
2 \mathrm{~mm} / 2 \mathrm{~mm}\end{array}$ & $\Delta \mathrm{WET}(\mathrm{mm})$ & $\begin{array}{l}\triangle \text { WET } \\
(\% \text { WET })\end{array}$ \\
\hline \multicolumn{5}{|c|}{ pCT versus refCT } \\
\hline \multirow[t]{2}{*}{0} & half & 38 & $9 \pm 4$ & $15 \pm 8$ \\
\hline & full & 16 & $17 \pm 8$ & $15 \pm 10$ \\
\hline \multirow[t]{2}{*}{90} & half & 14 & $7 \pm 5$ & $12 \pm 9$ \\
\hline & full & 8 & $17 \pm 8$ & $15 \pm 10$ \\
\hline \multirow[t]{3}{*}{180} & half & 13 & $9 \pm 7$ & $13 \pm 13$ \\
\hline & full & 16 & $17 \pm 8$ & $15 \pm 10$ \\
\hline & $\mathrm{vCT}_{\mathrm{T}}$ & is refCT & & \\
\hline \multirow[t]{2}{*}{0} & half & 100 & $-0.5 \pm 0.8$ & $-1.0 \pm 1.8$ \\
\hline & full & 98 & $-1.8 \pm 2.0$ & $-2.0 \pm 3.1$ \\
\hline \multirow[t]{2}{*}{90} & half & 99 & $-1.0 \pm 1.6$ & $-1.9 \pm 3.3$ \\
\hline & full & 98 & $-1.8 \pm 2.6$ & $-2.3 \pm 4.0$ \\
\hline \multirow[t]{2}{*}{180} & half & 98 & $-1.3 \pm 2.0$ & $-2.6 \pm 4.8$ \\
\hline & full & 98 & $-1.8 \pm 2.0$ & $-2.0 \pm 3.1$ \\
\hline
\end{tabular}

loss ring removal does not show in the $\mathrm{pCT}_{\mathrm{pCT}}$ versus refCT $_{\mathrm{pCT}}$ comparison. Larger deviations are observed for the $\mathrm{pCT}_{\mathrm{pCT}}$ versus $\mathrm{refCT}_{\mathrm{pCT}}$ at $180^{\circ}$ due to the different airway diameter. Generally the range differenced between the $\mathrm{vCT}_{\mathrm{pCT}}$ and $\mathrm{refCT}_{\mathrm{pCT}}$ are of the order of $2 \mathrm{~mm}$ or less.

Table 6 summarizes the results of the range comparison as well as the $D_{90}$ values for the target. The results are supporting the proposition that the $\mathrm{vCT}_{\mathrm{pCT}}$ is a surrogate for a replan-ning CT as $97 \%$ of pixels or more passed the distal range gamma evaluation. Values of $D_{90}$ between the $\mathrm{vCT}_{\mathrm{pCT}}$ and $\mathrm{refCT}_{\mathrm{pCT}}$ agreed well. The average range differences between vCT-pCT and refCT $_{\mathrm{pCT}}$ are found to be sub $\mathrm{mm}$ or below $1 \%$ of the range with a standard deviation or root mean square error of less than $2.1 \%$. When comparing the $\mathrm{pCT}_{\mathrm{pCT}}$ to the refCT $\mathrm{p}_{\mathrm{pCT}}$ we observed lower number of pixels passing the gamma evaluation as well as larger average range differences. While the radius of the phantom was reduced by $7 \mathrm{~mm}$, we did not observe this value in the mean range difference of table 6 at $0^{\circ}$ and $90^{\circ}$ as range was calculated from the phantom boundary. While these beams did miss the target in the $\mathrm{pCT}_{\mathrm{pCT}}$, as evidenced by the low $D_{90}$, their range was not necessarily modified by weight loss or neck tilt, as there were only slight inhomogeneities in the beam. The scenario was different for the $180^{\circ}$ beam which crossed an air cavity of different diameter in the $\mathrm{pCT}_{\mathrm{pCT}}$ and $\mathrm{refCT}_{\mathrm{pCT}}$. In that case we observed a larger range shift.

The better results of the range evaluation $(\sim 1 \% \pm 2 \%)$ compared to the WET evaluation $(\sim-2 \% \pm 3.5 \%)$ can be attributed to focus of the range evaluation on the central part of the phantom as opposed to the WET evaluation which covered the whole phantom with exception of the two outermost pixel rows. Additionally, the range is defined from the start of the phantom's surface down to the $80 \%$ isodose, which makes it less sensitive to misalignments of the phantom surface between two scans, as opposed to the WET. 

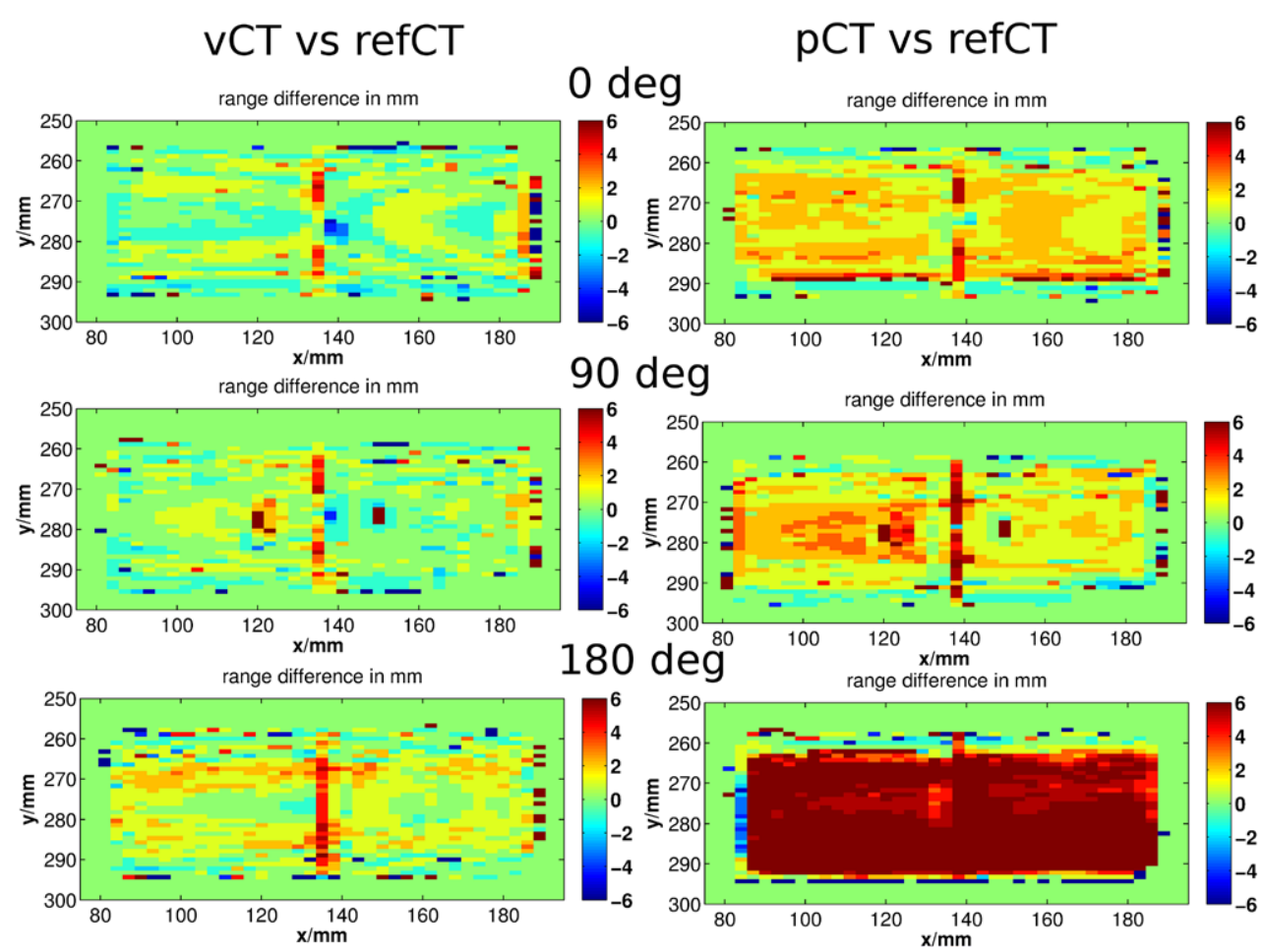

Figure 9. $\mathrm{BEV}$ range differences between (left) the $\mathrm{vCT}_{\mathrm{pCT}}$ and $\operatorname{refCT}_{\mathrm{pCT}}\left(\right.$ range $_{\mathrm{vCT}}$

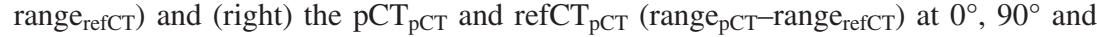
$180^{\circ}$. Range was calculated from the phantom surface to the $80 \%$ distal falloff.

Table 6. Results of the dosimetric evaluation comparing the $\mathrm{vCT}_{\mathrm{pCT}}$ to the refCT $\mathrm{T}_{\mathrm{pCT}}$ and the $\mathrm{pCT}_{\mathrm{pCT}}$ to the refCT $\mathrm{T}_{\mathrm{pCT}}$. $2 \mathrm{D}$ range maps are compared in terms of percent pixels passing the gamma test of $2 \mathrm{~mm} / 2 \mathrm{~mm}$ Range as well as the mean and standard deviation of range differences $\Delta \mathrm{R}$ ( range $_{\mathrm{vCT}}$ or pCTrange $_{\text {refCT }}$ ). The $D_{90}$ metric is compared as well.

\begin{tabular}{|c|c|c|c|c|c|c|c|c|c|}
\hline \multirow{2}{*}{$\begin{array}{l}\text { Angle } \\
\left({ }^{\circ}\right)\end{array}$} & \multirow{2}{*}{$\begin{array}{l}\text { components } \\
\text { traversed in } \\
\text { addition to } \\
\text { fat ring }\end{array}$} & \multicolumn{4}{|c|}{ vCT versus refCT } & \multicolumn{4}{|c|}{ pCT versus refCT } \\
\hline & & $\begin{array}{l}\% \\
\text { passing }\end{array}$ & $\Delta \mathrm{R}(\mathrm{mm})$ & $\Delta \mathrm{R}(\%)$ & $\begin{array}{l}\mathrm{D}_{90 \mathrm{vCT}} / \\
\mathrm{D}_{90 \mathrm{refCT}}\end{array}$ & $\begin{array}{l}\% \\
\text { passing }\end{array}$ & $\Delta \mathrm{R}(\mathrm{mm})$ & $\Delta \mathrm{R}(\%)$ & $\begin{array}{l}\mathrm{D}_{90 \mathrm{pCT}} / \\
\mathrm{D}_{90 \mathrm{refCT}}\end{array}$ \\
\hline 0 & muscle insert & 99 & $0.0 \pm 1.2$ & $0.0 \pm 1.7$ & 1.02 & 80 & $1.4 \pm 1.5$ & $1.8 \pm 2.0$ & 0.10 \\
\hline 90 & - & 97 & $0.2 \pm 1.6$ & $0.3 \pm 2.1$ & 1.00 & 82 & $1.3 \pm 2.0$ & $1.6 \pm 2.6$ & 0.65 \\
\hline 180 & airways & 97 & $0.7 \pm 1.4$ & $0.8 \pm 1.9$ & 0.99 & 29 & $6.7 \pm 4.4$ & $7.7 \pm 5.0$ & 0.95 \\
\hline
\end{tabular}

\subsection{Applicability of the method to a clinical dataset}

Qualitative assessment of the pCT to CBCT DIR for the patient case revealed no major dis-crepancy between the $\mathrm{vCT}_{\mathrm{CBCT}}$ and the CBCT. In particular, figure 10 presents results of the WET comparison using the gamma evaluation using criterion of $3 \mathrm{~mm} / 2$ $\mathrm{mm}$ WET. We observed that the percentage of pixels passing the gamma comparison to the $\mathrm{rpCT}_{\mathrm{CBCT}}$ is increased when using the $\mathrm{vCT}_{\mathrm{CBCT}}(94 \%)$ instead of the $\mathrm{pCT}_{\mathrm{CBCT}}$ $(77 \%)$. The mean WET 

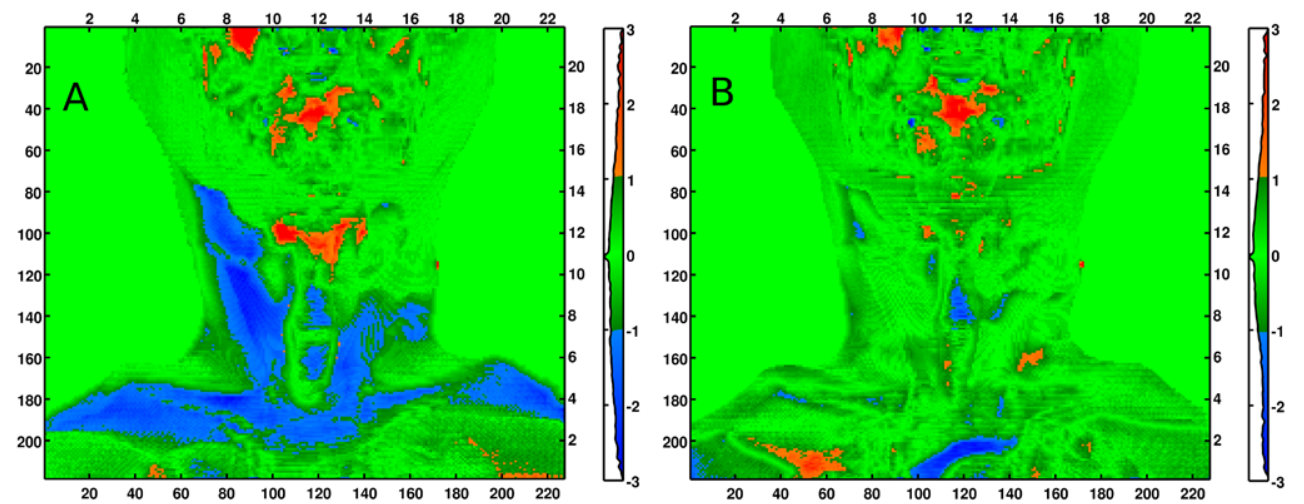

Figure 10. (a) Result of the gamma comparison of WET using criteria $3 \mathrm{~mm} / 2 \mathrm{~mm}$ WET of the $\mathrm{pCT}_{\mathrm{CBCT}}$ versus the $\operatorname{rpCT}_{\mathrm{CBCT}}\left(\mathrm{WET}_{\mathrm{pCT}}-\mathrm{WET}_{\mathrm{rpCT}}\right)$. (b) Comparison of the $\mathrm{vCT}_{\mathrm{CBCT}}$ to the $\mathrm{rpCT}_{\mathrm{CBCT}}\left(\mathrm{WET}_{\mathrm{vCT}}-\mathrm{WET}_{\mathrm{rpCT}}\right)$. The left and bottom axis are in pixels while the top and right axis are in $\mathrm{cm}$. The colorbar serves as a histogram of the gamma index distribution.

differences were $-0.6 \mathrm{~mm} \pm 3.2 \mathrm{~mm}$ and $1.1 \mathrm{~mm} \pm 3.6 \mathrm{~mm}$ respectively. The large neck portion failing the gamma evaluation for the $\mathrm{pCT}_{\mathrm{CBCT}}$ versus $\mathrm{rpCT}_{\mathrm{CBCT}}$ suggests that a beam coming from the left in figure 10 would suffer from range uncertainty, which could be reduced by employing the $\mathrm{vCT}_{\mathrm{CBCT}}$. A detailed analysis of DIR accuracy for clinical datasets is beyond the scope of this paper and will be investigated in a follow up study using rpCT scans as reference.

\section{Discussion}

In all investigated phantom configurations, automatically identified image features from the aSIFT algorithm suggest a geometrical deformation accuracy of the order of $2-3 \mathrm{~mm}$. We have confirmed that when using the Morphons algorithm CT numbers are preserved in homogeneous regions by the deformation, which is essential for accurate proton dose calculation. Our results indicate that for $\mathrm{H} \& \mathrm{~N}$-like cases the WET from the vCT agrees with a refCT to within $2 \mathrm{~mm}$, or about $2 \%-2.5 \%$ of the proton range. This was further supported by estimating the range from single beam IMPT dose distributions where maximum range differences of about $1 \mathrm{~mm}$ were observed corresponding to about $1 \%$ of the proton range. Additionally the $D_{90}$ obtained using the vCT was found to agree with the $D_{90}$ obtained using the refCT. This level of accuracy is acceptable when placed in the context of the range uncertainty from x-ray CT conversion, quoted as 3.5\% (Paganetti 2012). In fact, the final goal of our method is to provide a stopping power distribution allowing to flag clinical situations where the proton range has changed significantly. We do not aim to generate a stopping power distribution for direct IMPT re-planning; the latter should be performed based on images from the diagnostic CT scanner used for initial planning. In this envisioned application scenario the uncertainties we derived from our phantom study are acceptable.

This study has been focused on a phantom based evaluation of CT to CBCT DIR in the context of proton therapy. The use of a phantom allows the acquisition of a reliable reference image in treatment-day configuration, which is important for validation. While the validity of our approach for phantom data is a necessary condition for applicability to patient cases, it is not necessarily sufficient. Ideal patient-data based evaluation would rely on the acquisition of 
a CBCT and reference CT scan without patient re-positioning between scans. This is, however, difficult to achieve as in-room CT scanners are generally not installed along CBCT scanner. Furthermore it is unethical to scan patients twice as it violates the ALARA principle. The most likely patient datasets for evaluation would consist of daily or weekly CBCT images taken in parallel a few re-planning CT scans during treatment. Such a dataset would suffer from uncertainties from patient positioning at each scanner. Hence, these considerations support the use of a phantom for initial testing of the method; would it fail at this level there would be no need for further investigation.

Following the encouraging phantom results, in this work we have also applied our algorithm on a single patient case containing the data described above. We observed that the algorithm we tested on phantom data performed well on patient data. This supports further, thorough investigation of the method using several clinical datasets containing replanning CT and CBCT images, which is currently underway.

A limitation of our study is that our phantom fails to fully reproduce the range of patient size observed in H \& N CT images. In H \& N cancer cases adjuvant PTVs covering lymph nodes can extend into the upper thorax. In that region two issues are observed in CBCT images: i) a decrease of soft tissue CT number intensity due to increased beam hardening, scattering and attenuation from the shoulders and ii) truncation of the shoulders in the CBCT images due to the smaller FOV. Our initial patient results suggest that CT numbers on the vCT were not degraded by issue i). The use of an anterior beam to cover the region suffering from lateral truncation would also limit the impact of issue ii).

In-room CT would obviate the need for CBCT imaging as it directly provides diagnostic quality images comparable to those obtain from a planning CT scan. However in-room CT increases the complexity of both treatment rooms and workflows, as it entails couch movements between imaging and beam delivery. CBCT scanners can be integrated in a rotating gantry and require no couch shifts. An ideal alternative is to rely directly on the treatment beam to generate 3D stopping power maps from proton CT. While proton CT should provide more accurate stopping power distributions by avoiding the conversion of photon linear attenuation coefficients, it is not necessarily the ideal solution since it requires expensive beam time to generate images and no clinical device is yet available. In terms of dose calculation accuracy, the already mentioned range uncertainty from x-ray CT conversion is $3.5 \%$ and proton CT would most certainty reduce this. However, this level of uncertainty is most likely lower than uncertainties stemming from weight loss in $\mathrm{H} \& \mathrm{~N}$ cancer patients where range differences of several $\mathrm{mm}$ can easily be reached.

\section{Conclusion}

We have performed a thorough phantom evaluation of the possibility of deforming a pCT to a daily CBCT, thus creating a vCT, for proton dose recalculation. Applying the DIR workflow developed for the phantom to clinical data supported the validity of the method. In conclusion the vCT approach appears to be suitable for daily dose recalculation. In future work the validity of the $\mathrm{VCT}$ approach will be evaluated using more clinical $\mathrm{H} \& \mathrm{~N}$ patient data based on the tests employed in this study and by substituting the refCT by a re-planning CT scan.

\section{Acknowledgments}

This work was supported by the Federal Ministry of Education and Research of Germany (BMBF), grant number 01IB13001 (SPARTA) and by the German Research Foundation (DFG) 
Cluster of Excellence Munich-Centre for Advanced Photonics (MAP). Dr-Ing Johannes Wulz is acknowledged for designing the phantom. The authors would like to acknowledge Mark Podesta of MAASTRO clinic for sharing gamma evaluation code and Professor Dr Frank Verhaegen, Dr Brigitte Reniers and Dr Wouter van Elmpt for constructive discussions.

\section{References}

Agostinelli S et al 2003 GEANT4-a simulation toolkit Nucl. Instrum. Methods Phys. Res. A 506 250-303 Barker J L et al 2004 Quantification of volumetric and geometric changes occurring during fractionated radiotherapy for head-and-neck cancer using an integrated CT/linear accelerator system Int. J. Radiat. Oncol. Biol. Phys. 59 960-70

Bentefour E et al 2010 TU-A-204B-02: On the potential of CBCT for range verification in proton therapy Med. Phys. 373370

Cheung W and Hamarneh G 2009 n-SIFT: n-Dimensional scale invariant feature transform IEEE Trans. Image Process. 18 2012-21

Deasy J O et al 2003 CERR: a computational environment for radiotherapy research Med. Phys. 30 979-85

Janssens $\mathrm{G}$ et al 2009 Evaluation of nonrigid registration models for interfraction dose accumulation in radiotherapy Med. Phys. 36 4268-76

Janssens G et al 2011 Diffeomorphic registration of images with variable contrast enhancement J. Biomed. Imaging 2011 1-12

Kam M K et al 2007 Prospective randomized study of intensity-modulated radiotherapy on salivary gland function in early-stage nasopharyngeal carcinoma patients J. Clin. Oncol. 25 4873-9

Knutsson H and Andersson M 2005 Image Analysis ed H Kalviainen et al (Berlin: Springer) pp 292-301

Kraan A C et al 2013 Dose uncertainties in IMPT for oropharyngeal cancer in the presence of anatomical, range and setup errors Int. J. Radiat. Oncol. Biol. Phys. 87 888-96

Lowe D G 1999 Computer vision The Proc. of the Seventh IEEE Int. Conf. on (Kerkyra, 20-27 September 1999) vol 2 pp 1150-7

Lowe D 2004 Distinctive image features from scale-invariant keypoints Int. J. Comput. Vis. 60 91-110

Paganelli C et al 2013a Quantification of organ motion based on an adaptive image-based scale invariant feature method Med. Phys. 40111701

Paganelli C et al 2013b Scale invariant feature transform in adaptive radiation therapy: a tool for deformable image registration assessment and re-planning indication Phys. Med. Biol. 58 287-99

Paganetti H 2012 Range uncertainties in proton therapy and the role of Monte Carlo simulations Phys. Med. Biol. 57 R99-17

Park S et al 2009 On-board CBCT/CBDT for image-guided proton therapy: initial performance evaluation Int. J. Radiat. Oncol. Biol. Phys. 75 S595-S6

Peroni M et al 2012 Automatic segmentation and online virtual CT in head-and-neck adaptive radiation therapy Int. J. Radiat. Oncol. Biol. Phys. 84 e427-33

Persoon L C G G et al 2011 A fast 3D gamma evaluation using a GPU utilizing texture memory for on-the-fly interpolations Med. Phys. 38 4032-5

Schell S and Wilkens J J 2010 Advanced treatment planning methods for efficient radiation therapy with laser accelerated proton and ion beams Med. Phys. 37 5330-40

Shackleford J A et al 2010 On developing B-spline registration algorithms for multi-core processors Phys. Med. Biol. 55 6329-51

Simone C B 2nd et al 2011 Comparison of intensity-modulated radiotherapy, adaptive radiotherapy, proton radiotherapy and adaptive proton radiotherapy for treatment of locally advanced head and

neck cancer Radiother. Oncol. $101376-82$

Stock M et al 2009 Image quality and stability of image-guided radiotherapy (IGRT) devices: a comparative study Radiother. Oncol. J. Eur. Soc. Ther. Radiol. Oncol. 93 1-7

Trofimov A et al 2011 Interfractional variations in the setup of pelvic bony anatomy and soft tissue and their implications on the delivery of proton therapy for localized prostate cancer Int. J. Radiat.

Oncol. Biol. Phys. 80 928-37

van de Water T A et al 2011 Potential benefits of scanned intensity-modulated proton therapy versus advanced photon therapy with regard to sparing of the salivary glands in oropharyngeal cancer Int. J. Radiat. Oncol. Biol. Phys. 79 1216-24 
van Kranen S et al 2013 Adaptive radiotherapy with an average anatomy model: evaluation and quantification of residual deformations in head and neck cancer patients Radiother. Oncol. 109 463-8

Veiga C et al 2014 Toward adaptive radiotherapy for head and neck patients: feasibility study on using CT-to-CBCT deformable registration for 'dose of the day' calculations Med. Phys. 41031703

Wang W et al 2010 Clinical study of the necessity of replanning before the 25th fraction during the course of intensity-modulated radiotherapy for patients with nasopharyngeal carcinoma Int.

J. Radiat. Oncol. Biol. Phys. 77 617-21

Wrangsjo A et al 2005 Image Analysis ed H Kalviainen et al (Berlin: Springer) pp 501-10

Wu Q et al 2009 Adaptive replanning strategies accounting for shrinkage in head and neck IMRT Int.

J. Radiat. Oncol. Biol. Phys. 75 924-32

Yang Y et al 2007 Evaluation of on-board kV cone beam CT (CBCT)-based dose calculation Phys.

Med. Biol. 52 685-705

Zhang T et al 2007 Automatic delineation of on-line head-and-neck computed tomography images: toward on-line adaptive radiotherapy Int. J. Radiat. Oncol. Biol. Phys. 68 522-30

Zhen X et al 2012 CT to cone-beam CT deformable registration with simultaneous intensity correction Phys. Med. Biol. 57 6807-26 